\title{
Light-Induced Charge Transfer in Pyrene/CdSe-SWNT Hybrids**
}

\author{
By Liangbing Hu, Yan-Li Zhao, Koungmin Ryu, Chongwu Zhou, J. Fraser Stoddart, * \\ and George Grüner*
}

Dedicated to Professor David N. Reinhoudt on the occasion of his 65th birthday

On account of their nano-scale size, large aspect ratio and high conductivity, single-walled carbon nanotubes (SWNTs) have emerged as an attractive choice for conducting composite materials. ${ }^{[1-10]}$ Composites incorporating SWNTs show percolation dominated conductivity with a much lower volume threshold (volume fraction $\approx 10^{-5}$ ), compared to those with nanoparticles. ${ }^{[1-13]}$ Two-thirds of SWNTs are $p$-type semiconductors with holes as the charge carriers. Using the holeblocking nature of SWNTs, conducting polymers having SWNTs as dopants are effectively used as the hole buffering $^{[14]}$ and electron transport ${ }^{[15]}$ layers in organic light-emitted diodes (OLEDs). For the active layer of OLEDs and organic photovoltaic (OPV) devices, incorporation of SWNTs enhances the charge separation and facilitates charge transport, hence improving the performance, i.e., the short circuit current, the filling factor and power conversion efficiency. ${ }^{[16-19]}$ However, since SWNTs are a mixture of metallic and semiconducting nanotubes with a small bandgap $(\approx 0.6 \mathrm{eV})$, both electrons and holes in the composite matrix prefer ${ }^{[14]}$ to transfer onto and then be quenched on the SWNTs. Directly incor-

[*] Prof. J. F. Stoddart, Dr. Y.-L. Zhao[ï]

California NanoSystems Institute and

Department of Chemistry and Biochemistry

University of California, Los Angeles

405 Hilgard Avenue

Los Angeles, California 90095-1569 (USA)

E-mail: stoddart@chem.ucla.edu

Prof. G. Grüner, Dr. L. Hu ${ }^{[\dagger]]}$

Department of Physics and Astronomy

University of California, Los Angeles

405 Hilgard Avenue

Los Angeles, California 90095-1547 (USA)

E-mail: ggruner@ucla.edu

Dr. K. Ryu, Prof. C. Zhou

Department of Electrical Engineering

University of Southern California

University Park Campus

Los Angeles, California 90089 (USA)

[†] These authors contributed equally to this work.

[***] This work was supported by NSF Grant DMR-0404029, the Microelectronics Advanced Research Corporation (MARCO) and its Focus Center Research Program (FCRP) - Center on Functional Engineered Nano Architectonics (FENA), the Defense Advanced Research Projects Agency (DARPA), and the Center for Nanoscale Innovation for Defense (CNID). Supporting Information is available online at Wiley InterScience or from the author. porating SWNTs into active layers of OLEDs and OPV devices does not facilitate the electron/hole separation, nor does it improve the performances of devices. On the other hand, semiconductor nanoparticles, possessing large and tunable bandgaps (1-2 eV), can be incorporated into conducting polymers to form hybrid solar cells. ${ }^{[20-24]}$ Holes are transported along the polymer chains and electrons hop along the nanoparticle network. However, nanoparticles with large volume fractions are needed ${ }^{[22]}$ because of their high percolation threshold $(>\approx 30 \%)$. By contrast, the volume fraction for the conducting polymer is small, leading to low hole mobility and hence limiting the solar cell efficiency. Semiconductor nanorod-polymer hybrid solar cells benefit from the quasi-one-dimensional (1-D) electron transport along the rods, a mechanism which allows the use of a smaller volume fraction of nanorods and a larger volume fraction of polymer to conduct holes with higher mobilities. ${ }^{[25,26]}$ Although Alivisatos et al. ${ }^{[27-29]}$ have demonstrated that longer nanorods lead to higher energy conversion efficiency, nanorods of larger lengths and uniformly distributed diameters are difficult to fabricate. ${ }^{[30-32]}$

Semiconductor nanoparticle-SWNT hybrids have been the subject of recent interest as a consequence of the development of methods ${ }^{[33,34]}$ for the chemical modification of SWNTs. Such hybrids are well suited for use in optoelectronic devices, given the tunable bandgap of nanoparticles, quasione-dimensional (1-D) transport of SWNTs, and the ease of chemical fabrication. As part of a drive towards finding applications, an examination of the charge transfer (CT) between the various components of the hybrids is needed. By using such hybrid materials as photo-electrodes, efficient electron transfer from semiconductor nanoparticles, such as CdS, CdSe, and CdTe (donor) to SWNTs (acceptor) has been demonstrated by several groups ${ }^{[35-40]}$ to lead to increased photon generated current (Fig. 1). The CT also results in photolumi-

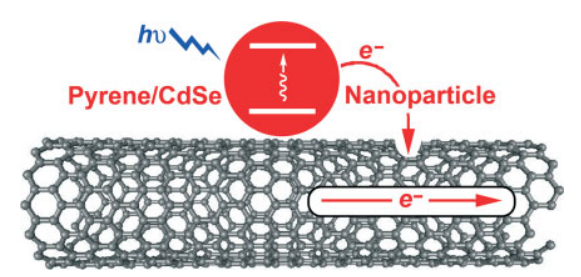

Figure 1. Schematic illustration of the light-induced charge excitation in a semiconductor nanoparticle and subsequent CT onto a SWNT, followed by charge transport along the SWNT. 
nescence quenching of semiconductor nanoparticle solutions. ${ }^{[38,39]}$ However, there is no work quantifying the magnitude of CT in the hybrids. SWNT field-effect transistors (FETs) have been widely used ${ }^{[7,41,42]}$ to study the CT from various analytes to SWNTs. In this communication, we use network transistors to study the CT from self-assembled pyrene-functionalized CdSe (pyrene/CdSe) nanoparticles on CVD-grown SWNTs and discuss the incorporation of such hybrids into OPV devices.

The surfaces of CdSe nanoparticles are functionalized ${ }^{[43-45]}$ with a pyrene derivative (see Supporting Information) of dihydrolipoic acid in $\mathrm{H}_{2} \mathrm{O} / \mathrm{N}, \mathrm{N}$-dimethylformamide (DMF) solution. The pyrene/CdSe nanoparticles were characterized (see Supporting Information) by ${ }^{1} \mathrm{H}$ NMR spectroscopy and transmission electron microscopy (TEM). The ${ }^{1} \mathrm{H}$ NMR spectra show characteristic proton resonances for the pyrene derivative and TEM images indicate that the average diameter of the pyrene/CdSe nanoparticles is $3.7 \mathrm{~nm}$ with a distribution of $1.2 \mathrm{~nm}$. The pyrene units attached to the surface of the CdSe nanoparticles associate with the surfaces of SWNTs by means of $\pi-\pi$ interactions to facilitate the formation of the pyrene/CdSe-SWNT hybrids. ${ }^{[38,46]}$ Solutions of the hybrids are prepared simply by adding SWNT powder into pyrene/CdSe nanoparticle solutions, followed by sonication at room temperature for eight hours. TEM images reveal that pyrene/ CdSe nanoparticles are attached randomly to the walls of SWNTs. TGA measurements indicate that the weight percentages of pyrene units in the pyrene/CdSe nanoparticles and the hybrids of SWNTs with pyrene/CdSe nanoparticles are ca. 38 and $20 \mathrm{wt} \%$, respectively. Therefore, the weight percentage of the pyrene/CdSe nanoparticles in the hybrids is ca. $53 \mathrm{wt} \%$.

In UV-vis experiments (see Supporting Information), gradual decreases of the absorption peak intensities of the pyrene/ CdSe nanoparticles $\left(0.10 \mathrm{~g} \mathrm{~L}^{-1}\right)$ around $380 \mathrm{~nm}$ (characteristic absorption peak of pyrene units) upon addition of SWNTs $\left(0-0.10 \mathrm{~g} \mathrm{~L}^{-1}\right)$ in DMF at $25^{\circ} \mathrm{C}$ indicate that the pyrene units become attached to the surfaces of SWNTs. The decrease in absorption results from the interaction between the pyrene/ CdSe nanoparticles and the SWNTs. ${ }^{[4-49]}$ We used $410 \mathrm{~nm}$ as the excitation wavelength $\left(\lambda_{\mathrm{ex}}\right)$ in all fluorescence experiments. The solutions of pyrene/CdSe nanoparticles $\left(0.1 \mathrm{~g} \mathrm{~L}^{-1}\right)$ and pyrene/CdSe-SWNT hybrids $\left(0.1 \mathrm{~g} \mathrm{~L}^{-1}\right)$ in DMF at room temperature show (Fig. 2a) orange yellow and dark brown colors, respectively. In the presence of light $\left(\lambda_{\mathrm{ex}}=410 \mathrm{~nm}\right)$, the pyrene/CdSe nanoparticle solution shows strong fluorescence but the hybrid solution reveals that fluorescent quenching is operative. The fluorescence intensity of the pyrene/ CdSe nanoparticle solution decreases significantly and the peak blue-shifts about $8 \mathrm{~nm}$ on the stepwise addition of a SWNT solution. Figure $2 \mathrm{~b}$ shows the changes in the fluorescence of the pyrene/CdSe nanoparticle solution $\left(0.15 \mathrm{~g} \mathrm{~L}^{-1}\right)$ upon addition of SWNTs in DMF (from $a$ to $j=0-0.2 \mathrm{~g} \mathrm{~L}^{-1}$ ) at $25{ }^{\circ} \mathrm{C}$. The strong dependence of quenching on the SWNT concentration implies that a CT process exists in the system. In such hybrid systems, the light generates electron-hole pairs.
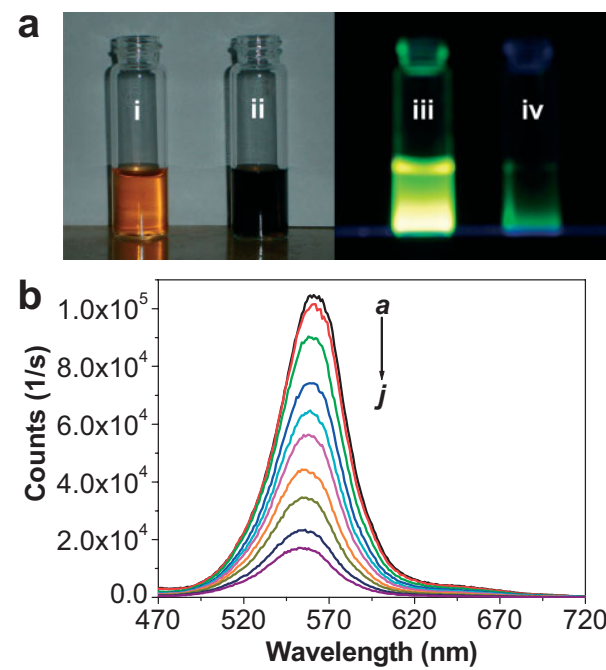

Figure 2. a) i) $0.1 \mathrm{~g} \mathrm{~L}^{-1}$ pyrene/CdSe nanoparticle in DMF solution. ii) $0.1 \mathrm{~g} \mathrm{~L}^{-1}$ pyrene/CdSe-SWNT hybrids in DMF solution. iii) Photoluminescence of the solution i) after excitation with $410 \mathrm{~nm}$ of light. iv) Quenched fluorescence of solution ii) after excitation with $410 \mathrm{~nm}$ of light. b) Changes in the fluorescence of the pyrene/CdSe nanoparticles $\left(0.15 \mathrm{~g} \mathrm{~L}^{-1}\right.$ ) upon addition of SWNTs (from $a$ to $j=0,0.01,0.02,0.05$, $0.08,0.10,0.12,0.15,0.18,0.20 \mathrm{~g} \mathrm{~L}^{-1}$ ) in DMF at $25^{\circ} \mathrm{C}, \lambda_{\text {ex }}=410 \mathrm{~nm}$.

The generated excitons tend to combine; meanwhile, the electrons or holes are transferred partially to the SWNTs, depending on the distance and the energy barriers between the pyrene/CdSe nanoparticles and the SWNTs. In other words, the formation of pyrene/CdSe-SWNT hybrids in solution favors the electrons or holes transfer from quantum dots (donor) to the SWNTs (acceptor), such that the exited electrons or holes enter the SWNTs, rather than be emitted.

In order to ascertain the direction of CT between the pyrene/CdSe nanoparticles and the SWNTs, the composite films were made by filtrating the solution through $100 \mathrm{~nm}$ alumina filter membranes. Figure 3 a shows the SEM image of the composite film, where the pyrene/CdSe nanoparticles are attached to the SWNTs. Silver paint contacts are made on the film with dimensions of $1 \times 1 \mathrm{~cm}^{2}$, and the resistance of the film is monitored by applying a $100 \mathrm{mV}$ bias voltage. The resistance increases when the light is turned on and decreases when the light is turned off (Fig. 3b), the light source being a light-emitting diode (LED) with an intensity $(I)$ of $40 \mathrm{~W} \mathrm{~m}^{-2}$ and excitation wavelength $\left(\lambda_{\mathrm{ex}}\right)$ of $410 \mathrm{~nm}$. The data have been fitted with an exponential formula, leading to time constants of 94 and $273 \mathrm{~s}$ for the increase and decrease, respectively. Since SWNTs are hole-transport materials, the increase of the resistance with the light on indicates that the direction of the electron transfer is from the nanoparticles to the SWNTs, a process which causes a decrease in hole concentration on the SWNTs. Later, we will learn that the time constant for the hybrids of self-assembled nanoparticles on the SWNTs is about $3.3 \mathrm{~s}$, which is much less than that of the composite, a situation which may be a result of the fact that the SWNTs are separated by the pyrene/CdSe nanoparticles in the com- 
a
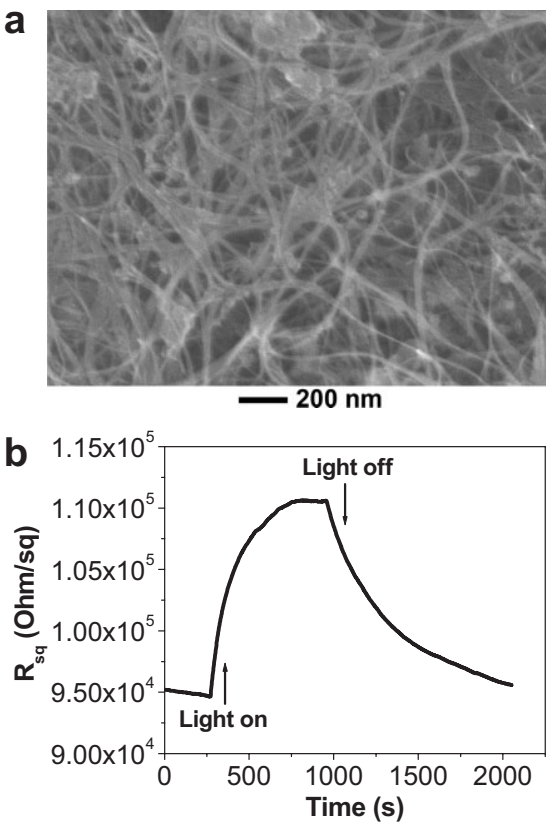

Figure 3. a) An SEM image of the pyrene/CdSe-SWNT composite on an alumina filter. b) Light-induced change of the resistance of a pyrene/ CdSe-SWNT composite film. I $=40 \mathrm{Wm}^{-2}$ and $\lambda=410 \mathrm{~nm}$. Assuming exponential time dependence, the time constants for the resistance in crease and decrease are $94 \mathrm{~s}$ and $273 \mathrm{~s}$, respectively.

posite film, leading to a longer time for reaching the charge equilibrium. The disparity between the large time constants found for the film (tens of seconds) and those found by others ${ }^{[40]}$ in the CdS-SWNT hybrid solution (several nanoseconds) is not yet understood.

In order to investigate the $\mathrm{CT}$ in the pyrene/CdSe-SWNT hybrids, FETs were fabricated using CVD-grown SWNT networks as the channels on silicon wafers with a $500 \mathrm{~nm}$ thick $\mathrm{SiO}_{2}$ dielectric. An AFM image (Fig. 4a) reveals that the average length of the SWNTs is approximately $2 \mu \mathrm{m}$ and the diameter is approximately $1.5 \mathrm{~nm}$. The density of the network was chosen to be 1.5 tubes $\mu \mathrm{m}^{-2}$ - just above the percolation threshold to avoid too many conduction paths through the metallic SWNTs, a situation which would reduce the on/off ratio of the transistor characteristics. ${ }^{[50,51]}$ The lift-off lithography process was used to pattern the $e$-beam-deposited Pd contact pads on top of the network of SWNTs. Oxygen plasma was used to etch away the SWNTs outside the source-drain channels. Since the channel is $200 \mu \mathrm{m}$ long and $1000 \mu \mathrm{m}$ wide - approximately 100 times longer than the SWNTs - the network, rather than the contact resistance between Pd and network, dominates the overall resistance. ${ }^{[50]}$ The pyrene/CdSe nanoparticles are self-assembled onto SWNT networks after the manner shown in Figure $4 \mathrm{~b}$. The silicon wafers with SWNT networks were soaked in pyrene/CdSe nanoparticle DMF solutions overnight, washed with $\mathrm{H}_{2} \mathrm{O}$ briefly, and blown dry with $\mathrm{N}_{2}$. We observed that the pyrene/CdSe nanoparticles become coated uniformly only onto the SWNT sur- faces and do not stick to the $\mathrm{SiO}_{2}$ surface (Fig. 4c). The height of the SWNTs after coating is approximately $5.0 \mathrm{~nm}$ (Fig. 4d), a distance which indicates that the height of the pyrene/CdSe nanoparticles is approximately $3.5 \mathrm{~nm}$. The nanoparticle size agrees well with the TEM data (see Supporting Information), a fact which indicates that the monolayer of pyrene/CdSe nanoparticles only becomes assembled onto one half of the surface of the SWNTs, the other half being directed towards the $\mathrm{SiO}_{2}$ surface. We tried drop-casting solutions of pyrene/ CdSe nanoparticles onto the source-drain channel of the devices and found that they shorted from source/drain to gate as a consequence of the leakage by pyrene/CdSe nanoparticles through the pinholes on the $\mathrm{SiO}_{2}$ dielectric. Devices fabricated by the self-assembly protocol do not exhibit a leakage problem, confirming our purposed mechanism that the surface-functionalized CdSe nanoparticles only assemble along the nanotubes.

The device configuration we have employed for resistance and transistor measurements is illustrated in Figure 5a. First, we measured the network resistance between the source and drain with zero-gate voltage. The resistance measurement was carried out by applying a $100 \mathrm{mV}$ bias with a Keithley 2400 apparatus: the data were recorded every $100 \mathrm{~ms}$. The network resistance responded quickly to the turning on and off of the LED. When the light was on, the sheet resistance increased and stayed high until the removal of the light. In order to eliminate the possibility that the change of the resistance was a result of the response of the SWNT network itself, we carried out a control experiment for the SWNT network without the coating of pyrene/CdSe nanoparticles and did not observe any detectable resistance change. A typical curve is plotted in Figure $5 \mathrm{~b}$, where $I=40 \mathrm{~W} \mathrm{~m}^{-2}$ and $\lambda=410 \mathrm{~nm}$. The data in the sharp increase region were enlarged and shown to fit well to an exponential equation, producing a time constant of $3.3 \mathrm{~s}$. Such a long time constant has also been observed for other SWNT hybrids, such as the SWNT-poly\{( $m$-phenylene-vinylene)-co-[(2,5-dioctyloxy- $p$-phenylene)]\} (PmPV) polymer ${ }^{[52]}$ with CdS nanoparticles, ${ }^{[40]}$ they are at least 10 times longer than the recently reported ${ }^{[53]}$ data for single crystal semiconductor nanoribbons. This long time constant, measured from the resistance, may not reflect the CT dynamics from the pyrene/CdSe nanoparticles to the SWNT, simply because of the fact that the charge reconfiguration over the entire network lasts until it reaches an equilibrium state. The same measurement on CdSe nanoparticles on an individual SWNT would shine light on the CT dynamics in the CdSe-SWNT hybrid.

The resistance change was found (Fig. 5c) to be a function of the light intensity where the excitation wavelength of the LED is $410 \mathrm{~nm}$. As the light intensity increases, the sheet resistance also increases and becomes saturated at $16 \mathrm{~W} \mathrm{~m}^{-2}$. Such a saturation has been observed in a SWNT-porphyrin hybrid system ${ }^{[54]}$ and for $\mathrm{CdSe}-\mathrm{TiO}_{2}{ }^{[21]}$ at light intensities of $40 \mathrm{~W} \mathrm{~m}^{-2}$ and $500 \mathrm{~W} \mathrm{~m}^{-2}$, respectively. The fact that the resistance change is a function of the light intensity indicates clearly that it is caused by the incident light on the hybrid net- 

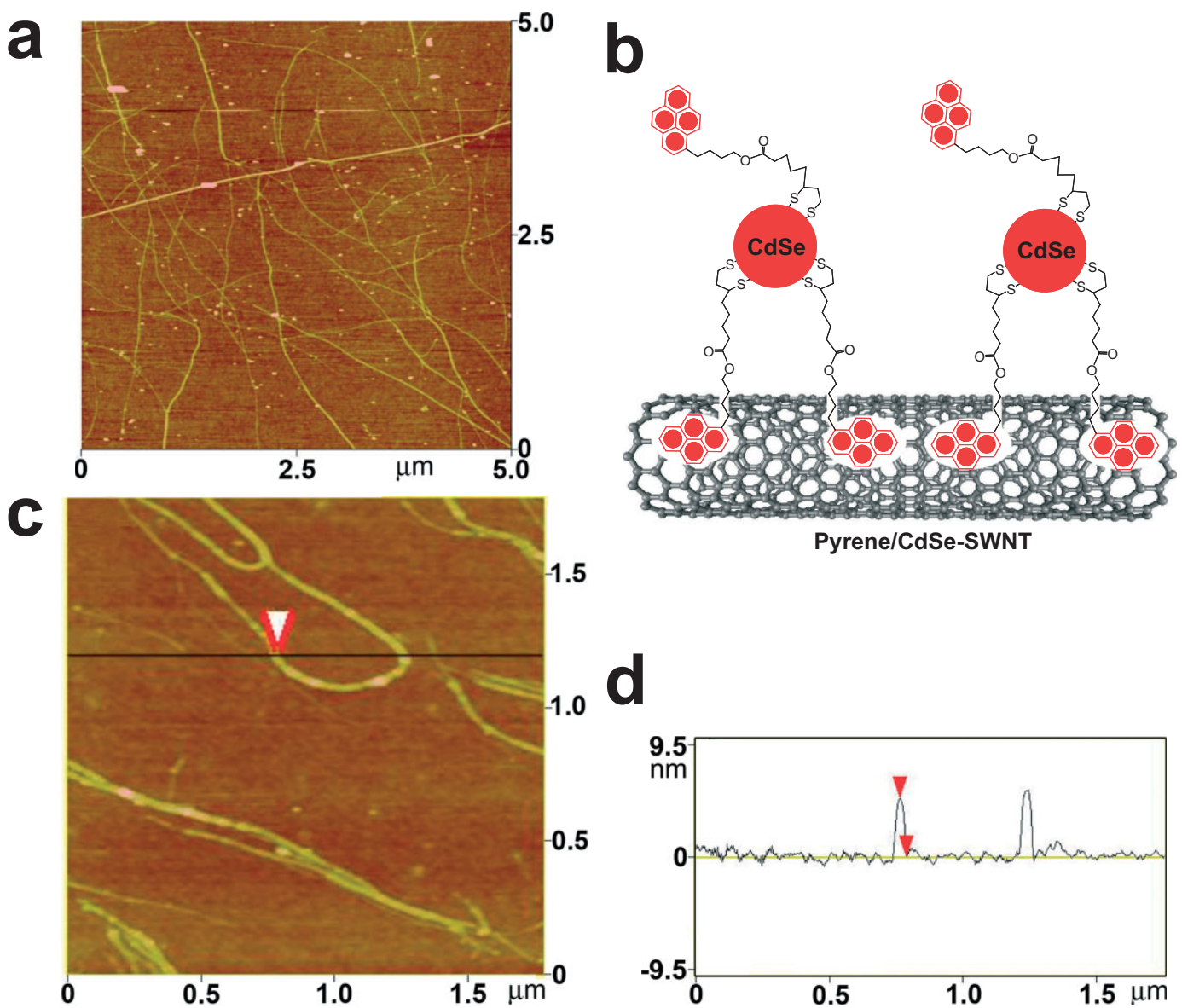

Pyrene/CdSe-SWNT

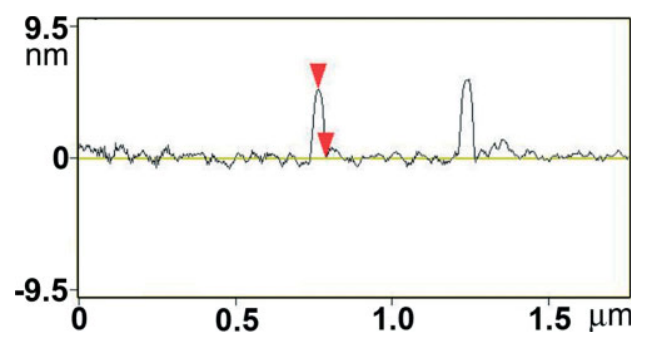

Figure 4. a) An AFM image of CVD-grown SWNTs with an average length of $2 \mu \mathrm{m}$ and a diameter of $1.5 \mathrm{~nm}$. b) Schematic drawing of the noncovalent bonding between surface-functionalized CdSe nanoparticles and a SWNT. c) An AFM image of self-assembled pyrene/CdSe nanoparticles on SWNTs. d) Cross section analysis of c). The height of the hybrid is approximately $5.0 \mathrm{~nm}$, indicating the average pyrene/CdSe nanoparticle size is approximately $3.5 \mathrm{~nm}$.

work. The CT dynamics in the network system is governed by the electron-hole pair generation, the CT onto the SWNTs, and the electron-hole recombination in the CdSe nanoparticles. As the light intensity increases, more excitons are generated. More electrons are transferred to the SWNTs and the resistance increases further. Meanwhile, the electron-hole pairs tend to combine and the electron-hole combination events increase as more excitons are generated. As the light reaches a particular intensity, an equilibrium state is attained and the saturation of the increase in the resistance is observed. The saturation of CT can help the optimization of the CdSe-SWNT hybrid OPV devices. The resistance change also shows (Fig. 5d) a strong dependence on the LED wavelength, provided the light intensity is $40 \mathrm{~W} \mathrm{~m}^{-2}$. When exciting the hybrid network with $585 \mathrm{~nm}$ LED, no detectable change in the resistance is observed. As the LED wavelength approaches the excitation wavelength $(410 \mathrm{~nm})$ of pyrene/CdSe nanoparticles, the change in the resistance increases. Although the excitation experiment shows emission only at $410 \mathrm{~nm}$, the sheet resistance changes when LEDs with other wavelengths, such as $470 \mathrm{~nm}$, are used. The response of the hybrid network to light with wavelengths other than the excitation wavelength is a consequence of the size distribution of the pyrene/CdSe nanoparticles (see Supporting Information). The bandgaps are proportional to the inverse square of the average nanoparticle size. The clear correlation between the change of sheet resistance and the intensity/wavelength of the light provide direct evidence that the light-induced change in the electronic structure of pyrene/CdSe nanoparticles is coupled with the CT through the SWNT network.

To study the CT from the pyrene/CdSe nanoparticles to the SWNTs in the FET, the transfer characteristic $\left(I_{\mathrm{sd}}\right.$ versus $\left.V_{\mathrm{g}}\right)$ of the transistor was measured by applying $100 \mathrm{mV}$ and sweeping the gate voltage between +20 and $-20 \mathrm{~V}$ in steps of $0.5 \mathrm{~V}$. Figure $6 \mathrm{a}$ shows the transfer curves of the SWNT network transistor before and after the assembly of the pyrene/ CdSe nanoparticles. The on and off currents decrease by a factor of 50 after the coating with the pyrene/CdSe nanoparticles, while the on/off ratio remains nearly unchanged (1877 and 1736, respectively). The calculated motilities are 

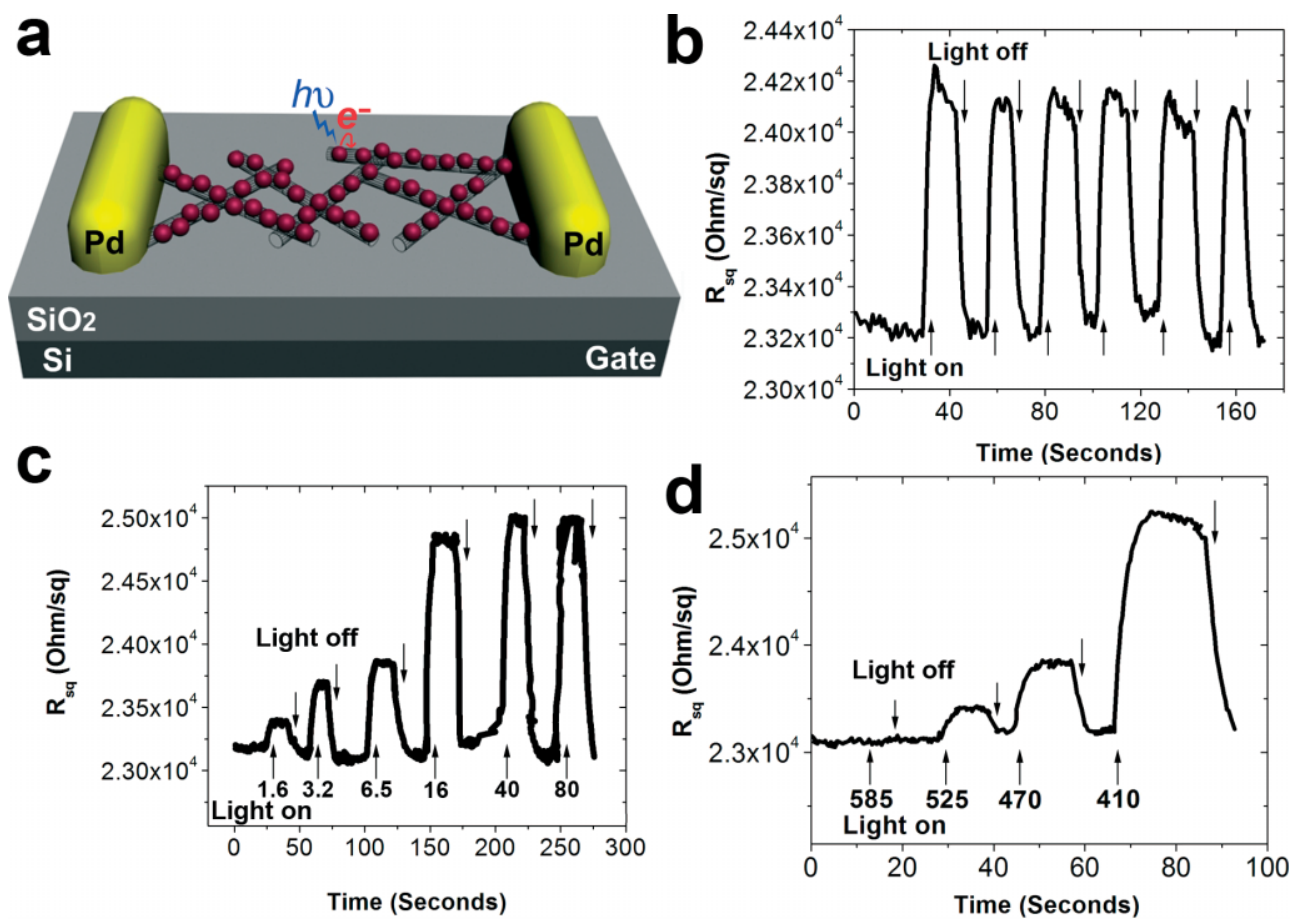

Figure 5. a) The schematic drawing of a SWNT FET with a coating of pyrene/CdSe nanoparticles on top of SWNTs. b) The resistance of a SWNT-FET device vs time monitored under zero gate voltage, $I=10 \mathrm{~W} \mathrm{~m}^{-2}$ and $\lambda=410 \mathrm{~nm}$. c) The resistance of the device vs time for different light intensities (I from 1.6 to $80 \mathrm{~W} \mathrm{~m}^{-2}$ ), where the LED excitation wavelength is $410 \mathrm{~nm}$ with a $15 \mathrm{~nm}$ width/half max. d) The resistance vs time for LEDs with different wavelengths, $\mathrm{I}=40 \mathrm{~W} \mathrm{~m}^{-2}$.
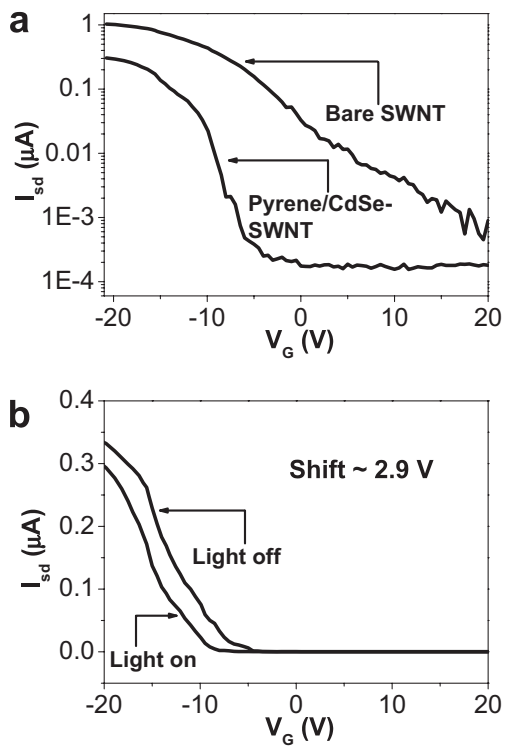

Figure 6. a) The transfer curve of the SWNT-FET before and after the assembly of pyrene/CdSe nanoparticle. The transistor curve after adding pyrene/CdSe nanoparticles both tilts and left-shifts, developments which indicate that the pyrene/CdSe nanoparticles introduce barriers to $\mathrm{CT}$ in the SWNT network and donate electrons onto the SWNTs. b) The transfer curves of the pyrene/CdSe-SWNT-FET when the light is off and on, $\lambda=410 \mathrm{~nm}$ and $\mathrm{I}=40 \mathrm{Wm}^{-2}$. The curve is horizontally shifted to the left after shining light on the network, a response which is a consequence of the CT from the pyrene/CdSe nanoparticles to the SWNTs when the light is switched on.
15.4 and $6.9 \mathrm{~cm}^{2} \mathrm{~s}^{-1} \mathrm{~V}^{-1}$, respectively. As a control, we tried to carry out the coating experiment of SWNTs with CdSe nanoparticles (Aldrich) in the FET and did not observe any appreciable change of transfer curve since the CdSe nanoparticles without the attached pyrene units are not immobilized on the surfaces of SWNTs under the same experimental conditions. The pyrene/CdSe nanoparticles assembled on top of the SWNTs have two effects. First, they act as charge scattering potentials and decrease the carrier mobility $(\mu)$ which is defined as the ratio of the slope of $I_{\mathrm{sd}}-V_{\mathrm{g}}$ curve to its initial slope. Second, the thermally excited electrons in pyrene/CdSe nanoparticles at room temperature are transferred to the SWNTs as the nanoparticles self-assemble onto SWNTs, a process which decreases the hole concentration (n) and shifts the threshold voltage of the transfer curve to the left. ${ }^{[42]}$ The current at zero gate voltage decreases by a factor of 200 after the assembly of the pyrene/CdSe nanoparticles. Since the tube-tube contact resistance is dominant in the SWNT network, such a large change in the resistance is, most likely, a consequence of the modification of the tube contact by the pyrene/CdSe nanoparticles. The operation voltage range from the on to off states decreases from 40 to $15 \mathrm{~V}$ in the measurement range after coating the devices with nanoparticles, a result which may be related to the modification of tube-tube contact by the pyrene/CdSe nanoparticles.

The transfer characteristics of the pyrene/CdSe-SWNT hybrid network with and without light $\left(40 \mathrm{~W} \mathrm{~m}^{-2}\right.$ intensity and 
$410 \mathrm{~nm}$ wavelength) are plotted in Figure 6b. Firstly, the SWNT-FET transfer curve was obtained in the dark. After the LED had been switched on for $10 \mathrm{~min}$, the transfer curve was measured again. Clearly, the transfer curve shifts to the left when the light is on without tilting. For the hybrid network, the scattering potential barriers caused by the pyrene/ CdSe nanoparticles remain unchanged when the light is on or off. The carrier mobility on the SWNTs does not depend on the electronic configuration of pyrene/CdSe nanoparticles. Therefore, no tilt of the transfer curve is observed. When the light is on, there are more electron-hole pairs in the pyrene/ CdSe nanoparticles besides the thermal excited pairs. Therefore, more electrons transfer from the pyrene/CdSe nanoparticles to the SWNTs, causing the transfer curve to shift further to the left by $2.9 \mathrm{~V}$. Together with the assumption of the fully coverage of the pyrene/CdSe nanoparticles on the SWNTs, a situation which is confirmed by the AFM image, the CT number per pyrene/CdSe nanoparticle can be calculated as follows. The total CT of the network is $C \times V$, where $C$ is the total capacitance of the SWNT network and $V$ is the shift value (here is $2.9 \mathrm{~V}$ ). $C$ is estimated from the total capacitance of each individual SWNT. The capacitance of each SWNT is calculated using the formula ${ }^{[55]} C=2 L \pi \varepsilon \varepsilon_{0} / \ln ((2 h / r)$ where $L \approx 2 \mu \mathrm{m}, h \approx 500 \mathrm{~nm}$, and $r \approx 1.5 \mathrm{~nm}$ for the SWNT and $\varepsilon \approx 4.1$ for $\mathrm{SiO}_{2}$. The SWNT density is $1.5 \mu \mathrm{m}^{2}$ and the size of the pyrene/CdSe nanoparticle is $3.7 \mathrm{~nm}$. We obtained the answer that the CT is 2.2 electron per pyrene/CdSe nanoparticle. This value corresponds to the maximum amount of CT since the light intensity was $40 \mathrm{~W} \mathrm{~m}^{-2}$, exceeding the saturation intensity of $16 \mathrm{~W} \mathrm{~m}^{-2}$. For comparison, previous studies ${ }^{[42,54]}$ on $\mathrm{NH}_{3}$ and porphyrin have revealed maximally 0.04 electrons per $\mathrm{NH}_{3}$ molecule and 0.37 electrons per porphyrin. This large amount of CT per pyrene/CdSe nanoparticle may be a result of the bigger size of the pyrene/CdSe nanoparticles compared to the porphyrin and $\mathrm{NH}_{3}$ molecules.

The effective CT from the pyrene/CdSe nanoparticles to SWNTs in the hybrid has the potential for applications in polymer hetero-junction photovoltaic devices. Currently, the highest efficiency of an organic solar cell ${ }^{[56]}$ is ca. $5 \%$ in poly(3-hexylthiophene) (P3HT) and 1-(3-methoxycarbonyl)propyl-1-phenyl[6,6] $\mathrm{C}_{61}(\mathrm{PCBM})$, although a $10 \%$ efficiency was predicted ${ }^{[25]}$ for such a system. In the P3HT-PCBM system, holes transport along the $\mathrm{P} 3 \mathrm{HT}$ polymer chains and electrons hop along the PCBM only. High weight percentage ${ }^{[57]}$ (optimally ca. $80 \%$ ) of the quasi-zero-dimensional (0-D) PCBMs is needed in the system in order to facilitate the electron transport along the PCBM. Therefore, one disadvantage of such systems is the low weight percentage for polymers, such as P3HT, to carry holes and hence the hole mobility is limited. Quasi-1-D objects, such as semiconductor nanocrystals, have been incorporated into conjugated OPVs to facilitate electron transport. ${ }^{[27-29]}$ The conductance close to the percolation threshold for the systems with quasi-0-D and quasi-1D objects are plotted in Figure 7a. For OPVs with quasi-0-D objects, the volume percolation threshold ${ }^{[58]}$ is $30 \%$. For ex-
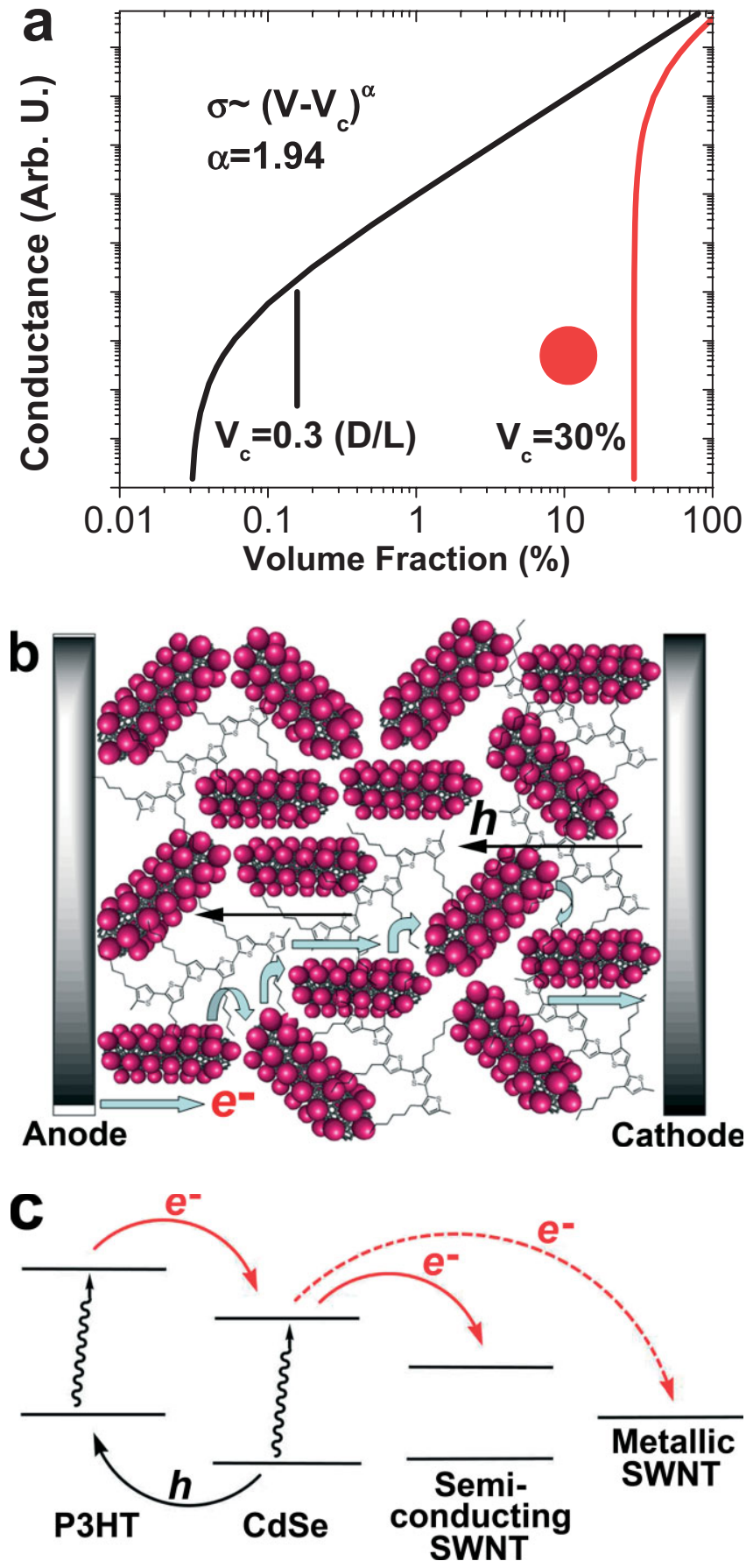

Figure 7. a) The dependence of the conductance for quasi-0-D objects (such as nanoparticles) and quasi-1-D objects (such as nanotubes) on the volume fraction near the percolation threshold. The composite with quasi-1-D objects (such as pyrene/CdSe-SWNT hybrids) has a much lower percolation threshold than that with quasi-0-D objects (such as nanoparticles). b) A schematic model for a hybrid OPV device incorporating a conducting polymer and pyrene/CdSe-SWNT hybrids, where the conducting polymer carries holes and the pyrene/CdSe-SWNT hybrids carry electrons. The SWNTs are fully covered by semiconductor nanoparticles. c) The energy diagram for the OPV device where light leads to the excitation of electron/holes in both the semiconductor nanoparticles and in the polymer. 
ample, the volume fraction of fullerene derivatives, such as PCBM in OPVs, must be larger than $30 \%$ in order that the electrons can be transported from the bulk to the electrodes. However, the volume threshold is much lower in composites with quasi-1-D objects, where it depends ${ }^{[59]}$ on the diameter (D) and the length ratio (L) (i.e., $0.3 \%$ for $\mathrm{D} / \mathrm{L}=0.01$ ). Here, we propose (Fig. 7b) an OPV structure with SWNTs covered by pyrene/CdSe nanoparticles. Both the polymers (such as $\mathrm{P} 3 \mathrm{HT}$ ) and the semiconductor nanoparticles absorb light in complementary ranges. For example, while P3HT absorbs strongly in the range of $450-600 \mathrm{~nm}$, which the size of semiconductor nanoparticles can be tuned to absorb light outside this range. Since the CT from the nanoparticle will saturate for certain light intensities (i.e., $16 \mathrm{~W} \mathrm{~m}^{-2}$ for CdSe in this work), it is necessary to incorporate hybrids using several nanoparticles with different bandgaps. The $\mathrm{D} / \mathrm{L}$ ratio is about 50 for $\mathrm{D}$ of $8 \mathrm{~nm}$ and $\mathrm{L}$ of $400 \mathrm{~nm}$, and the percolation threshold is $0.6 \%$ for hybrids carrying electrons to electrodes. We have designed solar cells with 50 volume percent of P3HT as the hole transport component and $50 \%$ semiconductor nanoparticle-SWNT hybrids for the electron transport component. The $50 \%$ electron transport component comprises different types of nanoparticles to cover the wavelength range, in a complementary fashion, with P3HT coverage. The CT dynamics are plotted in Figure 7c. The excited holes on the nanoparticles will transfer onto P3HT, just as in the $\mathrm{CdSe} /$ P3HT system and the electrons will transfer onto either metallic or semiconducting SWNTs. Solar cells incorporating the nanoparticle-SWNT hybrid will have advantages of (i) high electron/hole mobility, (ii) large exciton life time because of the effective charge separation from the nanoparticles to the SWNTs, and (iii) efficient light absorption. Recently, Raffaelle et al. ${ }^{[60]}$ have fabricated organic solar cells incorporating CdSe quantum dot-SWNT complexes. The devices, however, showed limited efficiency, a situation which is a result of the recombination of electrons and holes on the SWNTs since the nanoparticles only cover a small portion of the SWNT surface. ${ }^{[60]}$ In our proposed solar cells, it is essential that the SWNTs surface are fully covered by the nanoparticles for two reasons. Firstly, such hybrids can facilitate the electron transfer, while blocking hole transfer to the SWNTs, thus preventing electron/hole recombination because of the small bandgap associated with the SWNTs. Secondly, such hybrids will avoid the device short problem caused by the SWNTs. The SWNTs, which are micrometers long and highly conducting, can easily short the anode and cathode. By fully coating the SWNTs with pyrene/CdSe nanoparticles, the resistance between anode/cathode and the hybrid is large enough to avoid the short problem.

In conclusion, we have demonstrated effective CT from pyrene/CdSe nanoparticles to SWNTs by using both fluorescence quenching experiments and transistor measurements. The magnitude of the CT shows a strong dependency on the light intensity and wavelength and reaches a maximum of 2.2 electrons per pyrene/CdSe nanoparticle. The SWNTs act as electron acceptors and the pyrene/CdSe nanoparticles act as electron donors. On account of the small bandgaps of semiconducting SWNTs, the CT phenomena for semiconducting nanotubes will also be similar to those of metallic SWNTs. The next step will be to study the CT from the CdSe nanoparticles to the SWNTs with different chiralities by using individual SWNT transistors. In the present work, we only study the average effect of the CT from the pyrene/CdSe nanoparticles to SWNTs with different chiralities.

Furthermore, we have speculated on the incorporation of such acceptor-donor hybrids into organic solar cells in an attempt to improve the performance of the devices. Further studies on such unique hybrid systems are required. Examples include time-resolved fluorescence to probe the dynamics of the CT in solution, temperature dependent transport measurement to study the CT barrier between nanoparticles and SWNTs with different chiralities, as well as investigations of OPV devices incorporating fully covered SWNTs with semiconductor nanoparticles. Moreover, the methods we have used to study the CT and determine the magnitude of the CT can be applied to other semiconductor nanoparticle-SWNT hybrids. In the wake of our investigations on pyrene/CdSe nanoparticles, we will study fully the CT between the SWNTs and pyrene-modified semiconductor nanoparticles such as $\mathrm{CdS}, \mathrm{CdTe}$, and $\mathrm{CdSe} / \mathrm{CdS}$ and obtain their CT magnitudes from FET measurements. We will then be in a position to evaluate critically the role of CT in these semiconductor nanoparticle-SWNT hybrids.

\section{Experimental}

Experimental details are available in the Supporting Information.

Received: May 9, 2007

Revised: September 24, 2007 Published online: February 5, 2008

[1] M. Ouyang, J.-L. Huang, C. M. Lieber, Acc. Chem. Res. 2002, 35, 1018.

[2] H. Dai, Acc. Chem. Res. 2002, 35, 1035.

[3] D. M. Guldi, G. M. A. Rahman, F. Zerbetto, M. Prato, Acc. Chem. Res. 2005, 38, 871

[4] D. Tasis, N. Tagmatarchis, A. Bianco, M. Prato, Chem. Rev. 2006 106,1105

[5] Y.-P. Sun, K. Fu, Y. Lin, W. Huang, Acc. Chem. Res. 2002, 35, 1096.

[6] T. Ito, L. Sun, R. R. Henriquez, R. M. Crooks, Acc. Chem. Res. 2004, 37, 937.

[7] B. L. Allen, P. D. Kichambare, A. Star, Adv. Mater. 2007, 19, 1439.

[8] K. Keren, R. S. Berman, E. Buchstab, U. Sivan, E. Braun, Science 2003, 302, 1380.

[9] M. J. Biercuk, M. C. Llaguno, M. Radosavljevic, J. K. Hyun, A. T. Johnson, J. E. Fischer, Appl. Phys. Lett. 2002, 80, 2767.

[10] G. B. Blanchet, C. R. Fincher, F. Gao, Appl. Phys. Lett. 2003, 82, 1290.

[11] F. Du, J. E. Fischer, K. I. Winey, Phys. Rev. B 2005, 72, 121404.

[12] M. B. Bryning, M. F. Islam, J. M. Kikkawa, A. G. Yodh, Adv. Mater. 2005, 17, 1186.

[13] J. N. Coleman, S. Curran, A. B. Dalton, A. P. Davey, B. McCarthy, W. Blau, R. C. Barklie, Phys. Rev. B 1998, 58, 7492. 
[14] H. S. Woo, R. Czerw, S. Webster, D. L. Carroll, J. Ballato, A. E. Strevens, D. O'Brien, W. J. Blau, Appl. Phys. Lett. 2000, 77, 1393.

[15] P. Fournet, J. N. Coleman, B. Lahr, A. Drury, W. J. Blau, D. F. O’Brien, H.-H. Hörhold, J. Appl. Phys. 2001, 90, 969.

[16] E. Eymakis, G. A. J. Amaratunga, Appl. Phys. Lett. 2002, 80, 112.

[17] A. J. Miller, R. A. Hatton, S. R. P. Salva, Appl. Phys. Lett. 2006, 89, 123115.

[18] Z. Xu, Y. Wu, B. Hu, I. N. Ivanov, D. B. Geohegan, Appl. Phys. Lett. 2005, 87, 263118.

[19] E. Eymakis, I. Alexandrou, G. Amaratunga, Appl. Phys. Lett. 2003, 93, 1764.

[20] V. Aroutiounian, S. Petrosyan, A. Khachatryan, K. Touryan, J. Appl. Phys. 2001, 89, 2268.

[21] I. Robel, V. Subramanian, M. Kuno, P. V. Kamat, J. Am. Chem. Soc. 2006, 128,2385

[22] W. J. E. Beek, M. M. Wienk, R. A. J. Janssen, J. Mater. Chem. 2005, $15,2985$.

[23] T. Hasobe, H. Imahori, P. V. Kamat, S. Fukuzumi, J. Am. Chem. Soc. 2003, 125, 14962.

[24] W. J. E. Beek, M. M. Wienk, R. A. J. Janssen, Adv. Mater. 2004, 16, 1009.

[25] K. M. Coakley, M. D. McGehee, Chem. Mater. 2004, 16, 4533.

[26] H. Hoppe, N. S. Sariciftci, J. Mater. Res. 2004, 19, 1924.

[27] W. U. Huynh, J. J. Dittmer, A. P. Alivisatos, Science 2002, 295, 2425.

[28] W. U. Huynh, J. J. Dittmer, N. Teclemariam, D. J. Milliron, A. P. Alivisatos, K. W. Barnham, Phys. Rev. B. 2003, 67, 115326.

[29] D. J. Milliron, I. Gur, A. P. Alivisatos, MRS Bull. 2005, 30, 41.

[30] G. X. Wang, M. S. Park, H. K. Liu, D. Wexler, J. Chen, Appl. Phys. Lett. 2006, 88, 193115.

[31] T. Nann, J. Riegler, Chem. Eur. J. 2002, 8, 4791.

[32] G. R. Patzke, F. Krumeich, R. Nesper, Angew. Chem. Int. Ed. 2002, $41,2446$.

[33] J. Cao, J.-Z. Sun. J. Hong, H.-Y. Li, H.-Z. Chen, M. Wang, Adv. Mater. 2004, 16, 84 .

[34] Q. Li, B. Sun, I. A. Kinloch, D. Zhi, H. Sirringhaus, A. H. Windle, Chem. Mater. 2006, 18, 164.

[35] W. Li, C. Gao, H. Qian, J. Ren, D. Yan, J. Mater. Chem. 2006, 16, 1852.

[36] G. Mountrichas, S. Pispas, N. Tagmatarchis, Small 2007, 3, 404.

[37] S.-H. Hwang, C. N. Moorefield, P. Wang, K.-U. Jeong, S. Z. D. Cheng, K. K. Kotta, G. R. Newkome, J. Am. Chem. Soc. 2006, 128 , 7505.
[38] D. M. Guldi, G. M. A. Rahman, V. Sgobba, N. A. Kotov, D. Bonifazi, M. Prato, J. Am. Chem. Soc. 2006, 128, 2315.

[39] L. Sheeney-Haj-Ichia, B. Basnar, I. Willner, Angew. Chem. Int. Ed. $\mathbf{2 0 0 5}, 44,78$

[40] I. Robel, B. A. Bunker, P. V. Kamat, Adv. Mater. 2005, 17, 2458

[41] K. Bradley, M. Briman, A. Star, G. Grüner, Nano. Lett. 2004, 4, 253.

[42] K. Bradley, J.-C. P. Grabriel, M. Briman, A. Star, G. Grüner, Phys. Rev. Lett. 2003, 91, 218301.

[43] I. C. Gunsalus, L. S. Barton, W. Gruber, J. Am. Chem. Soc. 1956, 78, 1763.

[44] H. T. Uyeda, I. L. Medintz, J. K. Jaiswal, S. M. Simon, H. Mattoussi, J. Am. Chem. Soc. 2005, 127, 3870

[45] K. Palaniappan, C. Xue, G. Arumugam, S. A. Hackney, J. Liu, Chem Mater. 2006, 18, 1275.

[46] R. J. Chen, Y. Zhang, D. Wang, H. Dai, J. Am. Chem. Soc. 2001, 123 3838

[47] J. S. Kavakka, S. Heikkinen, I. Kilpeläinen, M. Mattila, H. Lipsanen, J. Helaja, Chem. Commun. 2007, 519.

[48] D. M. Guldi, G. M. A. Rahman, N. Jux, D. Balbinot, N. Tagmatarchis, M. Prato, Chem. Commun. 2005, 2038.

[49] A. Kongkanand, P. V. Kamat, ACS Nano 2007, 1, 13

[50] L. Hu, D. S. Hecht, G. Grüner, Nano Lett. 2004, 4, 2513.

[51] H. E. Unalan, G. Fanchini, A. Kanwal, A. Du Pasquier, M. Chhowalla, Nano Lett. 2006, 6, 677.

[52] A. Star, Y. Lu, K. Bradley, G. Grüner, Nano Lett. 2004, 4, 1587.

[53] J. S. Jie, W. J. Zhang, Y. Jiang, X. M. Meng, Y. Q. Li, S. T. Lee, Nano Lett. 2006, 6, 1887.

[54] D. S. Hecht, R. J. A. Ramirez, M. Brian, E. Artukovic, K. S. Chichak, J. F. Stoddart, G. Grüner, Nano Lett. 2006, 6, 2031

[55] T. Durkop, S. A. Getty, E. Cobas, M. S. Fuhrer, Nano Lett. 2004, 4, 35.

[56] G. Li, V. Shrotriya, J. Huang, Y. Yao, T. Moriarty, K. Emery, Y. Yang, Nature Mater. 2005, 4, 864 .

[57] G. Yu, J. Gao, J. C. Hummelen, F. Wudl, A. J. Heeger, Science 1995, $270,1789$.

[58] G. E. Pike, C. H. Seager, Phys. Rev. B 1974, 10, 1421.

[59] I. Balberg, N. Binenbaum, N. Wagner, Phys. Rev. Lett. 1984, 52, 1465.

[60] B. J. Landi, S. L. Castro, H. J. Ruf, C. M. Evans, S. G. Bailey, R. P. Raffaelle, Sol. Energ. Mater. Sol. Cell. 2005, 87, 733. 